\title{
Counting Binary Matrices with Given Row and Column Sums
}

\author{
By Ben Johnsen and Eldar Straume
}

\begin{abstract}
This paper is concerned with the calculation of certain numbers $s b(\bar{p}), b(\bar{p}, \bar{q})$ related to combinatorial problems and graph theory. $\bar{p}, \bar{q}$ are vectors of nonnegative integers, and $s b(\bar{p})$ is the number of labelled graphs with vertex degree sequence $\bar{p}$, or equivalently, the number of 0 -diagonal, symmetric, binary matrices with row sum $\bar{p}$. Similarly, $b(\bar{p}, \bar{q})$ is the number of binary rectangular matrices with row sum $\bar{p}$ and column sum $\bar{q}$. The numbers also appear as coefficients in the expansions $\Pi\left(1+x_{i} x_{j}\right), \Pi\left(1+x_{i} y_{j}\right)$.

Explicit (i.e., nonrecursive) formulas for $s b(\bar{p}), b(\bar{p}, \bar{q})$ are developed, together with an analysis of their complexity. Properties of $\bar{p}$ (or $\bar{q}$ ), such as $\max \left\{p_{i}\right\}, k=\sum p_{i}, n=$ $\#\left\{p_{i} \neq 0\right\}$, are incorporated into a numerical invariant which measures the total "cost" (or computing time). The performance of the theory for practical calculations has been thoroughly tested. For example, with suitable restrictions on $\bar{p}$ one may obtain $s b(\bar{p})$ for, say, $n=20$ or $k=30$.
\end{abstract}

0. Introduction. Let $\bar{p}=\left(p_{1}, p_{2}, \ldots, p_{n}\right)$ and $\bar{q}=\left(q_{1}, q_{2}, \ldots, q_{m}\right)$ be vectors of nonnegative integers. We are then interested in calculating two nonnegative integers $s b(\bar{p})$ and $b(\bar{p}, \bar{q})$ of certain combinatorial importance: $s b(\bar{p})$ is the number of labelled graphs with vertex degree sequence $\bar{p}$, or equivalently, the number of 0 -diagonal, symmetric, binary matrices with row sum $\bar{p}$. Similarly, $b(\bar{p}, \bar{q})$ is the number of binary rectangular matrices with row sum $\bar{p}$ and column sum $\bar{q}$. Algebraically, these numbers can be defined by simple polynomial identities, as follows. Let

$$
(x)=\left(x_{1}, x_{2}, \ldots, x_{n}\right), \quad(y)=\left(y_{1}, y_{2}, \ldots, y_{m}\right)
$$

be formal variables and

$$
\pi(x)^{\bar{p}}=x_{1}^{p_{1}} x_{2}^{p_{2}} \cdots x_{n}^{p_{n}}, \quad \pi(y)^{\bar{q}}=y_{1}^{q_{1}} y_{2}^{q_{2}} \cdots y_{m}^{q_{m}}
$$

monomials. Then the $s b$ - and $b$-numbers appear as coefficients in the expansions

$$
\begin{aligned}
& \prod_{i<j}\left(1+x_{i} x_{j}\right)=\sum s b(\bar{p}) \pi(x)^{\bar{p}}, \\
& \prod\left(1+x_{i} y_{j}\right)=\sum b(\bar{p}, \bar{q}) \pi(x)^{\bar{p}} \pi(y)^{\bar{q}} .
\end{aligned}
$$

The difficulties encountered in the calculations are due to the huge number of terms involved. For example, if $n=10$, there are $2^{45} \sim 10^{13}$ terms if the product in (0.1) is multiplied out. This also illustrates the practical problems in enumerative graph

Received November 23, 1981; revised June 23, 1986.

1980 Mathematics Subject Classification. Primary 05C30; Secondary 05-04, 05 A15. 
theory and clearly demonstrates the need for theoretical considerations before one embarks on (computer) calculations.

In the literature there are several contributions to the study of enumeration problems of the above type (see, e.g., Read [10], [11], [12], Snapper [17], Read and Wormald [13], [14]). Quite often, the representation theory of the symmetric group $S_{n}$ is involved, where $n$ is the "dimension" of the problem (cf. [17]). Sometimes one is content with a solution of closed formula type, for example, with the fact that a certain polynomial identity holds. However, in this case the problem of picking out coefficients of interest may still be formidable. When recursive procedures are used, solutions (or data) in dimension $n$ can be obtained once all the data in dimension less than $n$ are available. Thus, in some sense, this means one should calculate everything or nothing.

However, if one is only interested in a single, or more generally, a restricted class of monomials, a polynomial identity or a recursive formula may contain large quantities of redundant information which are hard to separate or which ultimately add up to, say, a coefficient known to be zero a priori. Therefore, an explicit formula for the $s b$ - (or $b$-) numbers, as developed in the present paper, cf. Theorem 3.1 (or 4.1), has many advantages:

- First, one can take advantage of all prior knowledge of monomials having the same coefficient. Call a relation $\sim$ on the set of monomials graphic if $\pi(x)^{\bar{p}} \sim$ $\pi(x)^{\bar{q}}$ implies $s b(\bar{p})=s b(\bar{q})$. It is then only necessary to calculate $s b(\bar{p})$ for one monomial in each equivalence class, preferably the monomial giving the simplest calculation.

- Secondly, many redundant terms can be eliminated. We shall work with ideals in the polynomial ring of monomials, for example, the ideal $I$ of those monomials not of interest (at present). Calculations modulo $I$ reduces the number of operations, terms to be stored, etc., since noncontributing quantities can be identified and eliminated at an early stage. This built-in flexibility allows extensions in one direction by putting restrictions in another. For example, in the case of $s b$-numbers, the dimension $n$ can be increased by restricting $\max p_{i}$ or $\sum p_{i}$.

- Thirdly, the explicit formula permits a detailed analysis of the complexity of the calculation of $s b(\bar{p})$ for any given $\bar{p}$. It is intuitively obvious that the complexity will increase with the number $n$ of vertices, the number $k$ of lines, the number of different partition numbers $p_{i}$ and the magnitude of these numbers. The numerical complexity formula of (5.1) explains precisely how these factors are related and the importance of each of them. In particular, this makes it possible to control the total complexity when some factors are increased by decreasing the others.

In all problems of this kind, numerical calculations will sooner or later be impracticable owing to the usual "exponential growth barrier". However, compared to a recursive procedure, the economy of the explicit formula seems to make it possible to push the calculations (far) into dimensions not previously available.

The paper is organized as follows. In Section 1 we define a graphic equivalence relation on the set of monomials, and in each class we identify a simple representative, called root monomial. Calculation of numbers $s b(\bar{p})$ only for a restricted class of monomials motivates the use of ideals, as described in Section 2. In this section we also recall some elementary results on symmetric functions, and certain polynomials, $\mu_{k}$, are defined by a simple standard construction. Here, $k=\frac{1}{2} \sum p_{i}$ is the 
number of edges when interpreted in the graph-theoretic sense. These polynomials, or rather their modified (truncated) versions $\mu_{k} \bmod I$, play a central role in the theory, since $s b(\bar{p})$ is expressed as a linear combination of their coefficients. Details are given in Section 3, and in Section 4 for the $b$-case, which has been developed along the sante lines as in Section 3. Finally, in Section 5 we look at the results obtained with possible applications in mind. This requires some theoretical considerations such as those leading to a formula for the numerical complexity of monomials. The latter is in fact roughly proportional to the computing time needed for $s b(\bar{p})$.

The enumeration of unlabelled graphs with given vertex degree sequence $\left(p_{1}, p_{2}, \ldots, p_{n}\right), n \leqslant 10$, has been completed by Read and Wormald [13], [14] by recursive methods (with respect to $n$ ). Our method, restricted to the calculation of $s b$-numbers for $n \leqslant 10$, will give the "labelled" part of their work. Since the calculations in this paper are based upon an explicit formula for the $s b$-numbers, this nonrecursive method gives coefficients of individual monomials. The unlabelled case will be treated in a separate paper. Restrictions on $\sum p_{i}$ or max $p_{i}$, rather than $n$, will permit us to extend previous results in enumerative graph theory.

1. Root Monomials. In this section we shall introduce a graphic relation on the set of monomials and choose a representative, called root monomial, from each equivalence class. Our choice will be justified by the fact that it is easy to perform and, most important, is expected to give the simplest calculation of the $s b$ - (or $b$-) number of the class. With the notation from Section 0 , the monomials $\pi(x)^{\bar{p}}$ and $\pi(x)^{\bar{p}} \pi(y)^{\bar{q}}$ will be called $s b$ - and $b$-monomial, respectively. An equivalence relation on the set of monomials (of fixed type) is called graphic if $s b(\bar{p})$ (or $b(\bar{p}, \bar{q})$, resp.) is constant on each class. We shall now describe one such relation, $\sim$.

First, take one class consisting of all monomials whose $s b$-number (or $b$-number, resp.) is zero. All other monomials are called graphic, and are characterized by the following results:

1.1. TheOREM (Erdös and Gallai [2]). The monomial $\pi(x)^{\bar{p}}$, with $p_{1} \geqslant p_{2} \geqslant \cdots \geqslant$ $p_{n}$ and $\sum p_{i}$ even, is graphic if and only if

$$
\sum_{\alpha=1}^{k} p_{\alpha} \leqslant k(k-1)+\sum_{\alpha=k+1}^{n} \min \left\{k, p_{\alpha}\right\} \quad \text { for all } 1 \leqslant k \leqslant n .
$$

1.2. TheORem (Gale, Ryser, cf. [16, p. 63]). The monomial $\pi(x)^{\bar{p}} \pi(y)^{\bar{q}}$, with $p_{1} \geqslant \cdots \geqslant p_{n}, q_{1} \geqslant \cdots \geqslant q_{m}$ and $\sum p_{i}=\sum q_{i}$, is graphic if and only if

$$
\sum_{\alpha=1}^{k} q_{\alpha} \leqslant \sum_{\alpha=1}^{k} \#\left\{l \mid p_{l} \geqslant \alpha\right\} \text { for all } 1 \leqslant k \leqslant m .
$$

Using these two theorems, we can easily decide if a given monomial is graphic or not; we turn to the description of $\sim$ on the set of graphic monomials.

We shall define a duality/contraction operator ${ }^{*}$ on monomials, motivated by corresponding operations on binary matrices. If $f$ is a 0 -diagonal, symmetric, binary $n$-matrix with row sum $\bar{p}$, or a binary $n \times m$-matrix with row sum $\bar{p}$ and column sum $\bar{q}$, we associate with it the monomial

$$
\omega(f)=\pi(x)^{\bar{p}}, \quad \text { or } \pi(x)^{\bar{p}} \pi(y)^{\bar{q}},
$$

respectively. Then $(0.1),(0.2)$ are equivalent to

$$
s b(\bar{p})=\# \omega^{-1}\left(\pi(x)^{\bar{p}}\right), \quad b(\bar{p}, \bar{q})=\# \omega^{-1}\left(\pi(x)^{\bar{p}} \pi(y)^{\bar{q}}\right) .
$$


Since vectors $\bar{p}, \bar{q}$ may have zero components, put

$$
N(\bar{p})=\#\left\{\alpha \mid p_{\alpha}>0\right\}, \quad K(\bar{p})=\sum p_{\alpha} .
$$

The action of $*$ on $\bar{p}$ will depend only on the $N(\bar{p})$ nonzero components of $\bar{p}$ up to a permutation; thus assume $p_{1} \geqslant p_{2} \geqslant \cdots$, and define in the $s b$-case

$$
*(\bar{p})=\left(N(\bar{p})-1-p_{N(\bar{p})}, N(\bar{p})-1-p_{N(\bar{p})-1}, \ldots\right),
$$

where all zero components are deleted. With $\bar{p}$ interpreted as vertex degree sequence of a graph with $N(\bar{p})$ vertices, the operation ${ }^{*} \pi(x)^{\bar{p}}=\pi(x)^{*(\bar{p})}$ corresponds to taking the dual graph and deleting isolated vertices. In the $b$-case we are led to the operation ${ }^{*}\left(\pi(x)^{\bar{p}} \pi(y)^{\bar{q}}\right)=\pi(x)^{*(\bar{p})} \pi(y)^{*(\bar{q})}$ via the correspondence (1), where ${ }^{*}(\bar{p}),{ }^{*}(\bar{q})$ are defined as in (3), except that $N(\bar{p})-1-p_{i}$ must be replaced by $N(\bar{q})-1-p_{i}$, and $N(\bar{q})-1-q_{i}$ by $N(\bar{p})-1-q_{i}$, respectively. Now, let $\sim$ on graphic $s b$-monomials be the smallest equivalence relation such that

$$
\begin{aligned}
& \text { (a) } \pi(x)^{\bar{p}} \sim \pi(y)^{\bar{r}} \text { if } \bar{r} \text { is a permutation of } \bar{p}, \\
& \text { (b) } \pi(x)^{\bar{p}} \sim^{*} \pi(x)^{\bar{p}} .
\end{aligned}
$$

Similarly, in the $b$-case we choose $\sim$ such that

$$
\begin{aligned}
\pi(x)^{\bar{p}} \pi(y)^{\bar{q}} & \sim \pi(x)^{\bar{r}} \pi(y)^{\bar{s}} \sim *\left(\pi(x)^{\bar{p}} \pi(y)^{\bar{q}}\right) \\
& \sim \pi(x)^{\bar{q}} \pi(y)^{\bar{p}},
\end{aligned}
$$

where $\bar{r}, \bar{s}$ is a permutation of $\bar{p}, \bar{q}$, respectively.

By establishing bijections like

$$
\left\{f \mid \omega(f)=\pi(x)^{\bar{p}}\right\} \leftrightarrow\left\{f \mid \omega(f)={ }^{*} \pi(x)^{\bar{p}}\right\},
$$

the following theorem is easily verified.

\subsection{THEOREM. The equivalence relation $\sim$ is a graphic relation.}

Which representative of a class should be chosen as root monomial? As explained in Section 0, we would like to minimize the numerical complexity of $\pi(x)^{\bar{p}}$ according to formula (5.1). This number will increase with $N(\bar{p}), K(\bar{p}), \max \left\{p_{i}\right\}$ and some other characteristics of $\bar{p}$ (see Sections 0 and 5), and the operator * tends to decrease these numbers until the process stabilizes when

$$
\left.* *(\bar{p})=\bar{p} \quad \text { (assume } p_{1} \geqslant p_{2} \geqslant \cdots\right) \text {. }
$$

1.4. THEOREM. In each equivalence class of graphic monomials $\pi(x)^{\bar{p}}$ there is a unique element $\pi(x)^{\bar{r}}$ such that

(a) ${ }^{* *}(\bar{r})=\bar{r}$

(b) $K(\bar{r}) \leqslant K(*(\bar{r}))(c f .(2))$.

Moreover, if equality holds, then we add the following condition:

(c) $\bar{r} \leqslant *(\bar{r})$ in the lexicographic order.

Clearly, $\bar{r}$ is obtained from $\bar{p}$ by applying * a finite number of times until (a) holds, indicating that $\pi(x)^{\bar{r}}$ or $\pi(x)^{*(\bar{r})}$ should be taken as root monomial of the class. Then we simply use (b), (c) to make the final choice among $\bar{r},{ }^{*}(\bar{r})$. However, this procedure may fail to give smallest numerical complexity, although in most cases the latter is reasonably minimized, cf. Section 5. For $b$-monomials, an algorithm for the choice of root monomials, leading to the simplest calculation of $b(\bar{p}, \bar{q})$ in Section 4 , can be constructed as in Theorem 1.4 , but we omit details here. 
The number of equivalence classes of graphic $s b$-monomials in dimensions $\leqslant 12$ are listed in the last row of Table I, Section 2. Moreover, a list of root monomials (in certain dimensions) and the corresponding $s b$-numbers is given in Johnsen and Straume [5].

2. The Algebraic Setting. The algebraic calculations are considered formally to take place in the polynomial ring $\mathbf{Q}\left[x_{1}, x_{2}, \ldots\right]$ or its quotient $\bmod I$, where $I$ is an ideal containing the monomials not of interest. The effect of $I$ during calculations is to identify and get rid of monomials which will not contribute to the final result. Such ideals arise naturally in situations where one wants to keep the magnitude of a characteristic feature low.

In fact, let $\delta$ be a function on the vectors $\bar{p}$ with values in the rational numbers such that $\delta(\bar{p}) \geqslant \delta(\bar{q})$ if $p_{i} \geqslant q_{i}$ for all $i$. Then if $d$ is a fixed number, the monomials $\left\{\pi(x)^{\bar{p}} \mid \delta(\bar{p}) \geqslant d\right\}$ are the monomials of an ideal. Typical such functions are

(1) $K(\bar{p})=\sum p_{i}$,

(2) $\delta(\bar{p})=\max \left\{p_{i}\right\}$,

(3) $\delta_{d}(\bar{p})=\#\left\{i \mid p_{i}>d\right\}$,

(4) $\delta_{i}(\bar{p})=p_{i}$,

(5) $\delta_{i, j}(\bar{p})=p_{i}+p_{j}$.

Since ideals can be combined by the usual algebraic rules, it is clear that various types of restrictions can be described by ideals in this way.

In order to calculate, say $s b(\bar{p})$, for a single specific $\bar{p}$, one should choose the largest convenient ideal $I(\bar{p})$ not containing $\pi(x)^{\bar{p}}$, e.g., $\pi(x)^{\bar{q}} \in I(\bar{p})$ if $q_{i}>p_{i}$ for some $i$. However, to make the later calculations of interest for all monomials, we shall stick to ideals of type

$$
I(d)=\left\{\pi(x)^{\bar{p}} \mid \max \left\{p_{i}\right\}>d\right\},
$$

where $d$ is a positive integer. The definition of numerical complexity of $\pi(x)^{\bar{p}}$ in Section 5 will take the ideal $I\left(\max \left\{p_{i}\right\}\right)$ into account, and before embarking on calculations, it is worth checking if the monomial $\pi(x)^{\bar{p}}$ can be replaced by one with lower complexity. Note that no graphic sb-monomial $\pi(x)^{\bar{p}}$ with $N(\bar{p}) \leqslant n$ is contained in $I(n-1)$, hence calculations in $\mathbf{Q}\left[x_{1}, x_{2}, \ldots, x_{n}\right]$ may always be reduced $\bmod I(n-1)$, and even $\bmod I(n-2)$ if $\pi(x)^{\bar{p}}$ is replaced by a suitable monomial equivalent to it.

Table I gives the number of equivalence classes of graphic $s b$-monomials having a representative $\notin I$ and with lowest dimensional monomial (in the class) of $\operatorname{dim} n$. The last row gives the number of root monomials of $\operatorname{dim} n(I=0)$, and $I=I(d)$ in the other rows.

For the sake of convenience and easy reference we shall fix some notations and recall properties of symmetric functions. Let

$$
\begin{array}{ll}
(z)=\left(z_{1}, z_{2}, \ldots, z_{m}\right), & t_{l}=\sum_{i} z_{i}^{l}, \quad l=1,2, \ldots, \\
(x)=\left(x_{1}, x_{2}, \ldots, x_{n}\right), & s_{l}=\sum_{i} x_{i}^{l}, \quad l=1,2, \ldots,
\end{array}
$$




\section{TABLE I}

The number of graphic sb-equivalence classes.

\begin{tabular}{|c|r|r|r|r|c|c|r|r|r|r|r||r|r|r|r|r|}
\hline$d r^{n}$ & 2 & 3 & 4 & 5 & 6 & 7 & 8 & 9 & 10 & 11 & 12 & 16 & 17 & 18 & 19 & 20 \\
\hline 3 & 0 & 0 & 2 & 6 & 14 & 16 & 25 & 25 & 36 & 36 & 49 & 81 & 81 & 100 & 100 & 121 \\
4 & 0 & 0 & 2 & 6 & 20 & 49 & 83 & 110 & 146 & 182 & 231 & 489 & 570 & 670 & 770 & 891 \\
5 & 0 & 0 & 2 & 6 & 20 & 69 & 202 & 336 & 504 & 672 & 924 & 2445 & 2970 & 3685 & 4400 & 5346 \\
\hline$n-2$ & 0 & 0 & 2 & 6 & 20 & 69 & 269 & 981 & 3647 & 13658 & 51766 & & & & & \\
\hline
\end{tabular}

be formal variables and corresponding power sums. Applying the identification (or substitution)

$$
(z)=\left(x_{1} x_{2}, x_{1} x_{3}, \ldots, x_{1} x_{n}, x_{2} x_{3}, \ldots, x_{n-1} x_{n}\right),
$$

i.e., $m=\left(\begin{array}{l}n \\ 2\end{array}\right)$, we get

$$
t_{l}=\frac{1}{2}\left(s_{l}^{2}-s_{2 l}\right), \quad l=1,2, \ldots
$$

Now, let $\tau_{k}$ be the $k$ th elementary symmetric function, so that

$$
\prod_{i=1}^{m}\left(1+z_{i}\right)=\sum_{k=0}^{m} \tau_{k}(z) .
$$

It is a classical result that $\tau_{k}(z)$ can be expressed as a polynomial in the power sums $t_{j}$. In particular, there are polynomials satisfying

$$
\tau_{k}\left(z_{1}, z_{2}, \ldots, z_{m}\right)=\lambda_{k}\left(t_{1}, t_{2}, \ldots, t_{k}\right)=\mu_{k}\left(s_{1}, s_{2}, \ldots, s_{2 k}\right),
$$

where $\mu_{k}$ is derived from $\lambda_{k}$ via (2). Recursive formulas are

$$
k \lambda_{k}=\sum_{l=1}^{k}(-1)^{l+1} t_{l} \lambda_{k-l}, \quad k \geqslant 1 ; \lambda_{0}=1 .
$$

From (3) we see that the modified polynomials $\bar{\lambda}_{k}=k ! \lambda_{k}$ and $\bar{\mu}_{k}=k ! 2^{k} \mu_{k}$ have integer coefficients, and moreover, that

$$
\bar{\mu}_{k}=\sum_{l=1}^{k}(-1)^{l+1}(k-1) \cdots(k-l+1) 2^{l-1}\left(s_{l}^{2}-s_{2 l}\right) \bar{\mu}_{k-l} .
$$

These formulas are not suitable for getting information about coefficients, their magnitude or the number of terms included. Since we need this information explicitly, the method explained below is used for calculating $\bar{\mu}_{k}$ on a computer.

Use the notation for coefficients explained by the expansions

$$
\lambda_{k}(t)=\sum_{\bar{r}} \lambda_{k}(\bar{r}) \pi(t)^{\bar{r}}=\mu_{k}(s)=\sum_{\bar{q}} \mu_{k}(\bar{q}) \pi(s)^{\bar{q}} .
$$

Then there is the well-known formula

$$
\lambda_{k}(\bar{r})=\left\{\begin{array}{cl}
\frac{(-1)^{k+\Sigma r_{i}}}{1^{r_{1}} 2^{r_{2}} \cdots k^{r_{k}} r_{1} ! r_{2} ! \cdots r_{k} !} & \text { if } \sum i r_{i}=k, \\
0 & \text { otherwise, }
\end{array}\right.
$$

so $\mu_{k}(\bar{q})$ can be found by applying the substitution (2) to $\pi(t)^{\bar{r}}$ in (5). Technically, this amounts to expanding $t_{l}^{r_{l}}=\left(\frac{1}{2}\left(s_{l}^{2}-s_{2 l}\right)\right)^{r_{l}}$ and determining coefficients $t(\bar{r}, \bar{q})$ such that

$$
\pi(t)^{\bar{r}}=\sum_{\bar{q}}(-1)^{\sum q_{i}} t(\bar{r}, \bar{q}) \pi(s)^{\bar{q}} .
$$


In this summation, however, the space of vectors $\bar{q}$ is unknown, but the calculations can be performed according to the following result, which is easily verified.

2.6. Proposition. Let $\bar{r}$ be given, $\sum i r_{i}=k$. Then

$$
\pi(t)^{\bar{r}}=\sum_{\bar{u}, \bar{v}}(-1)^{\sum q_{i}} t(\bar{r}, \bar{q}) \pi(s)^{\bar{q}},
$$

where $\bar{u}, \bar{v}$ runs through all (nonnegative integral) vectors such that $\bar{u}+\bar{v}=\bar{r}$, $\bar{q}=2 \bar{u}+\bar{w}, \bar{w}=\left(0, v_{1}, 0, v_{2}, \ldots\right)$, and moreover

$$
t(\bar{r}, \bar{q})=\frac{r_{1} ! r_{2} ! \cdots r_{k} !}{2^{\sum r_{i}} u_{1} ! u_{2} ! \cdots v_{1} ! v_{2} ! \cdots} .
$$

2.7. Remarks. (a) Note that the $\mu_{k}$-polynomials are calculated from the $\lambda_{k}$-polynomials by a standard "Wreath product" type of substitution, called "substitution of polynomials" by Read [12]. Except for the sign of coefficients, $\lambda_{k}$ is identical to the cycle index polynomials $Z\left(S_{k}\right)$ of the symmetric group $S_{k}$. For $k \leqslant 10$ a table of $Z\left(S_{k}\right)$ is given in Harary and Palmer [4, p. 249].

(b) Of course, it is a simple matter to generate all partitions $\bar{r}$ of $k\left(\sum i r_{i}=k\right)$, and calculate the integer coefficients $k ! \lambda_{k}(\bar{r})$ of $\bar{\lambda}_{k}$. Then, for each $\bar{r}$ one generates terms $\pi(s)^{\bar{q}}$ of $\bar{\mu}_{k}$ as in Proposition 2.6, and so the coefficients of $\bar{\mu}_{k}$ are calculated cumulatively, since different partitions $\bar{r}$ may contribute to the coefficient of the same term. However, it will be evident later that in order to keep the number of terms reasonably small as $k$ increases, which in turn will increase the speed of further calculations in Sections 3 and 4, we are only interested in mod $I$ versions of the above polynomials. This modification is explained below. (For the number of terms, see Table II, Section 5 , for $k \leqslant 30$.)

Now, fix $k$ and let $I \subset \mathbf{Q}\left[x_{1} x_{2}, \ldots\right]$ be an ideal. We may always assume $I$ contains all monomials $\pi(x)^{\bar{p}}$ with $\sum p_{i}>2 k$ or $\max \left\{p_{i}\right\}>k$. We would like to get rid of those terms in $\mu_{k}(s)$ which belong to $I$ when $(s)$ is expressed by $(x)$, cf. (2.1). First, let $\lambda_{k}^{I}(t)$ be the truncation of $\lambda_{k}(t)$ obtained by putting $\lambda_{k}(\bar{r})=0$ if $t_{l}=\sum_{i<j} x_{i}^{l} x_{j}^{l} \equiv 0$ for some $l \leqslant k$ such that $r_{l}>0$. Similarly, put $t(\bar{r}, \bar{q})=0$ in (7) if for some $l, s_{l} \equiv 0, q_{l}>0$. This is the case, for example, if $q_{l}>0$ for some $l>k$. Next, use the modified versions of $\lambda_{k}(s), t(\bar{r}, \bar{q})$ to calculate a polynomial $\mu_{k}^{I}(s)$ as before. Note that $\mu_{k}^{I}(s)$ has only $k$ variables $\left(s_{1}, s_{2}, \ldots, s_{k}\right)$, it is congruent to both $\mu_{k}\left(s_{1}, \ldots, s_{2 k}\right)$ and $\mu_{k}\left(s_{1}, \ldots, s_{k}, 0, \ldots, 0\right) \bmod I$, but $\mu_{k}^{I}$ is not in general equal to the last polynomial. Define polynomials for $l \leqslant k$ by

$$
\mu_{k}(s) \bmod I(l)=\mu_{k}\left(s_{1}, \ldots, s_{l}, 0, \ldots, 0\right) .
$$

These are the candidates used for $\mu_{k}$ in the calculations, since they are congruent to $\mu_{k}^{I(l)} \bmod I(l)$ and congruent polynomials will do the same job. In the next section we show how these polynomials enter into the calculation of $s b$-numbers.

3. Calculation of $s b$-numbers. We shall present a method for the calculation of $s b$-numbers which seems to be sufficiently different from those previously studied to be of some interest. By definition, $s b(\bar{p})$ is the coefficient of $\pi(x)^{\bar{p}}$ in $\Pi\left(1+x_{i} x_{j}\right)$, so from (2.3), (2.4) the identities

$$
\sum s b(\bar{p}) \pi(x)^{\bar{p}}=\sum \tau_{j}(z)=\sum \lambda_{j}(t)=\sum \mu_{j}(s)
$$


hold if the variables $(x),(z),(t),(s)$ are related as in (2.1), (2.2). If $I$ is an ideal as in Section 2, and

$$
T_{k}^{I}=\tau_{k}(z) \bmod I=\mu_{k}(s) \bmod I=\sum s b(\bar{p}) \pi(x)^{\bar{p}}
$$

denotes the sum over monomials $w=\pi(x)^{\bar{p}}$ with fixed $K(\bar{p})=\sum p_{i}=2 k$ and $w \notin I$, we have the recursive formula of integer coefficient polynomials

$$
k ! T_{k}^{I} \equiv \sum_{l}(-1)^{l+1}(k-1) \cdots(k-l+1) t_{l}(k-l) ! T_{k-l}^{I} \bmod I
$$

with $t_{l}=\sum_{i<j} x_{i}^{l} x_{j}^{l} \bmod I$.

The remainder of this section is devoted to getting an explicit (nonrecursive) formula for the computation of $s b(\bar{p})$ for a single graphic (root) monomial $w$, or a certain class $A=\left\{w_{i}\right\}$ of such monomials with some "similarity." We assume $K(\bar{p})=2 k$ is fixed for all $w \in A$, and let $I$ be an ideal (as large as we conveniently can find) such that $A \cap I=\varnothing$. In particular, $I$ contains all $\pi(x)^{\bar{p}}$ with $K(\bar{p})>2 k$ or $\max \left\{p_{i}\right\}>k$. Note that $\mu_{k}(s)$ in (2) can be any polynomial $\equiv \mu_{k}^{I}(s) \bmod I$, so if we have generated such a polynomial, as explained in Section $2, s b(\bar{p})$ will occur as the coefficient of $\pi(x)^{\bar{p}}$ when $(s)$ is expressed by $(x)$. Hence, if $S(\bar{r}, \bar{l})$ is the coefficient defined by

$$
\pi(s)^{i}=\sum_{\bar{r}} S(\bar{r}, \bar{l}) \pi(x)^{\bar{r}}
$$

then clearly

$$
s b(\bar{p})=\sum_{i} \mu_{k}^{I}(\bar{l}) S(\bar{p}, \bar{l}),
$$

where $\mu_{k}^{I}(\bar{l})$ is the coefficient of $\pi(s)^{l}$ in the polynomial $\mu_{k}^{I}$.

$S(\bar{p}, \bar{l})$ in (5) is obtained by an expansion of power sum products into monomials. This is a classical problem; see, for instance, Riordan [15] and the references given there. For us, however, it is important to get a formula expressed by $\bar{p}, \bar{l}$, which simplifies considerably if $\bar{p}$ contains equal components. Such a formula is developed below.

With ideal $I \subset \mathbf{Q}\left[x_{1}, \ldots, x_{n}\right]$ as before, let $m=m(I)=\max \left\{j \mid s_{j} \not \equiv 0\right\}$. As usual, all integers encountered are nonnegative and vectors are integral. The vector $\bar{b}=\left(b_{1}, \ldots, b_{n}\right)$ is called a ( )-partition (of dimension $n$ ) of $l$ if $\sum b_{i}=l$. The vector $[c]=\left[c_{1}, c_{2}, \ldots, c_{m}\right]$ is called a [ ]-partition (of dimension $m$ ) of $p$ if $\sum i c_{i}=p$. Thus, if $(u)$ is an $m \times n$-matrix of integers, then row $i$ is a ( )-partition of $\sum_{j} u_{i j}=l_{i}$ and column $j$ is a [ ]-partition of $\sum_{i} i u_{i j}=p_{j}$. Let $M(\bar{l}, \bar{p})$ be the set of all such matrices, for given $\bar{p}=\left(p_{1}, \ldots, p_{n}\right), \bar{l}=\left(l_{1}, \ldots, l_{m}\right)$. Using the multinomial formula on $\left(s_{j}\right)^{l_{j}}$, it is readily seen that

$$
S(\bar{p}, \bar{l})=\left(\prod l_{j} !\right) \sum \frac{1}{\prod u_{i j} !}
$$

with summation over all $(u) \in M(\bar{l}, \bar{p})$.

However, the summation space $M(\bar{l}, \bar{p})$ in (6) tends to be quite large for many choices of $\bar{p}$, so we propose the following modification for the calculation of $S(\bar{p}, \bar{l})$. Let $p_{m}\left(p_{i}\right)$ be the number of [ ]-partitions of $p_{i}$ (of dimension $m$ ), written as column vectors, and let

$$
\left(\left[p_{i}\right]\right)=\left(\left[p_{i}\right]_{1},\left[p_{i}\right]_{2}, \ldots,\left[p_{i}\right]_{h_{m}\left(p_{i}\right)}\right)
$$


be the ordered set of these vectors. Write

$$
\bar{p}=(\overbrace{p_{1}, \ldots,}^{r_{1}} \overbrace{p_{2}, \ldots,}^{r_{2}} p_{3}, \ldots, \ldots, \overbrace{p_{t}, \ldots, p_{t}}^{r_{t}}),
$$

where $p_{1}>p_{2}>\cdots>p_{t}>0$ and the first $r_{1}$ components of $\bar{p}$ are equal, the next $r_{2}$ are equal, etc. By definition, any matrix $(u) \in M(\bar{l}, \bar{p})$ has the first $r_{1}$ columns from the set $\left(\left[p_{1}\right]\right)$, the next $r_{2}$ columns from $\left(\left[p_{2}\right]\right)$, etc. Furthermore, the first $r_{1}$ columns will consist of $r_{11}$ columns equal to [ $\left.p_{1}\right]_{1}, r_{12}$ columns equal to [ $\left.p_{1}\right]_{2}$, etc., such that the vector $\left(r_{11}, r_{12}, \ldots, r_{1 p_{m}\left(p_{1}\right)}\right)$ describes the first $r_{1}$ columns up to order. These can be arranged in

$$
Q_{1}=\left(\begin{array}{c}
r_{1} \\
r_{11} r_{12} \cdots r_{1 p_{m}\left(p_{1}\right)}
\end{array}\right)
$$

ways. With similar notations for the next $r_{2}$ columns, etc., the array

$$
R=\left(\begin{array}{c}
r_{11} \cdots r_{1 p_{m}\left(p_{1}\right)} \\
\hdashline r_{t 1} \cdots r_{t p_{m}\left(p_{t}\right)}
\end{array}\right)
$$

determines $Q(R)=Q_{1} Q_{2} \cdots Q_{l}$ matrices $(u)=(u(R))$ in $M(\bar{l}(R), \bar{p})$ with the same row sum vector $l(R)$ and moreover, the same value of $\Pi(u(R)) !=\prod u_{i j}$ !. In fact,

$$
\begin{gathered}
l(R)=r_{11}\left[p_{1}\right]_{1}+r_{12}\left[p_{1}\right]_{2}+\cdots+r_{t p_{m}\left(p_{t}\right)}\left[p_{t}\right]_{p_{m}\left(p_{t}\right)}, \\
\prod(u(R)) !=\left(\prod\left[p_{1}\right]_{1} !\right)^{r_{11}}\left(\prod\left[p_{1}\right]_{2} !\right)^{r_{12}} \cdots\left(\prod\left[p_{t}\right]_{p_{m}\left(p_{t}\right)} !\right)^{r_{t p_{m}\left(p_{t}\right)}} .
\end{gathered}
$$

In this notation, (6) reads

$$
S(\bar{p}, \bar{l})=\sum_{R} \frac{\prod \bar{l}(R) ! Q(R)}{\Pi(u(R)) !}
$$

with summation over the set $M(\bar{l})$ of all arrays $R$ such that $\bar{l}=\bar{l}(R)$ and row $i$ is a ( )-partition of $r_{i}$ of dimension $h_{m}\left(p_{i}\right)$. Combining this with (5), we have the following result.

3.1. TheOREM. Let $I$ be an ideal not containing $\pi(x)^{\bar{p}}, k=\frac{1}{2} \sum p_{i}$. Then, with the above notation,

$$
s b(\bar{p})=\sum_{R} \frac{\prod \bar{l}(R) ! Q(R) \mu_{k}^{I}(\bar{l}(R))}{\Pi(u(R)) !}
$$

with summation over all arrays $R$, as in (7), where row $i$ is $a$ ( )-partition of $r_{i}$ of dimension $p_{m}\left(p_{i}\right)$.

4. Calculation of $b$-numbers. In this section we show how to calculate the number of $n \times m$-dimensional binary matrices with row sum $\bar{p}$ and column sum $\bar{q}$. This is the coefficient $b(\bar{p}, \bar{q})$ of the monomial $w=x_{1}^{p_{1}} x_{2}^{p_{2}} \cdots x_{n}^{p_{n}} y_{1}^{q_{1}} y_{2}^{q_{2}} \cdots y_{m}^{q_{m}}$ in (0.2). As pointed out by Kerber [7, p. 119], this number also appears in the enumeration of double cosets of the symmetric group $S_{k}$. The classical Gale-Ryser theorem only tells us when $b(\bar{p}, \bar{q})>0$. One method of calculating $b$-numbers, which utilizes the character table of $S_{k}, k=\sum p_{i}$, is given in Snapper [17]. The method described below is closely connected with the calculation of $s b$-numbers in Section 3 , and the formula for $b(\bar{p}, \bar{q})$ is analogous to that of Theorem 3.1. The idea is to keep the (column sum) vector $\bar{q}$ fixed and calculate $b(\bar{p}, \bar{q})$ for various $\bar{p}$. 
Let $I$ be an ideal in $\mathrm{Q}\left[x_{1}, x_{2}, \ldots, x_{n}\right]$ not containing monomials $\pi(x)^{\bar{p}}$ of interest. It is easily seen that

$$
\tau_{q_{1}}(x) \cdots \tau_{q_{m}}(x) \equiv \sum b(\bar{p}, \bar{q}) \pi(x)^{\bar{p}} \bmod I
$$

with summation over all $\bar{p}$ such that $\pi(x)^{\bar{p}} \pi(y)^{\bar{q}}$ is graphic and $\pi(x)^{\bar{p}} \notin I$. Hence, given $\pi(x)^{\bar{p}}$, the problem is to pick out its coefficient in the expansion (1).

Define a mod $I$ version of the polynomial $\lambda_{k}$, as in Section 2, namely

$$
\lambda_{k}^{I}(s)=\sum_{\bar{r}} \lambda_{k}^{I}(\bar{r}) \pi(s)^{\bar{r}}
$$

where

$$
\lambda_{k}^{I}(\bar{r})= \begin{cases}\lambda_{k}(\bar{r}) & \text { if } r_{j}=0 \text { for all } j \text { such that } s_{j} \equiv 0 \\ 0 & \text { otherwise }\end{cases}
$$

and $s_{j}=x_{1}^{j}+\cdots+x_{n}^{j}$. From (2.4),

$$
\tau_{q_{1}}(x) \cdots \tau_{q_{m}}(x) \equiv \lambda_{q_{1}}^{I}(s) \cdots \lambda_{q_{m}}^{I}(s) .
$$

Denote the polynomial on the right side of (2) by

$$
\mu_{\bar{q}}^{I}(s)=\sum \mu_{\bar{q}}^{I}(\bar{l}) \pi(s)^{l}
$$

it has coefficients

$$
\mu_{\bar{q}}^{I}(\bar{l})=\sum \lambda_{q_{1}}^{I}\left(\bar{v}_{1}\right) \cdots \lambda_{q_{m}}^{I}\left(\bar{v}_{m}\right)
$$

with summation over all $m(I) \times m$-matrices $(v)$ in $M(\bar{l}, \bar{q})$ with column vectors $\bar{v}_{i}$. Here $m(I)=\max \left\{j \mid s_{j} \not \equiv 0\right\}$, and the set $M(\bar{l}, \bar{q})$ is defined in Section 3. Thus, the polynomial (3) can be calculated by using the explicit values for the factors in (4) given by (2.5). Formula (4) is Theorem 1 in Levine [8], and referring to MacMahon [9, pp. 45-46], Levine gives some hints on how to evaluate this formula in practical cases. As in Section 3, the summation set $M(\bar{l}, \bar{q})$ can be reduced (with the same motivation, since the case of equal $p$ 's is equally interesting here) to a set $M(\bar{l})$ as in Section 3. Using $\mu_{\bar{q}}^{I}(s)$ instead of $\mu_{k}^{I}(s)$ in Section 3, we get the analogous result.

4.1. THEOREM. Let $\pi(x)^{\bar{p}} \pi(y)^{\bar{q}}$ be a graphic monomial and I any ideal in $\mathbf{Q}\left[x_{1}, \ldots, x_{n}\right]$ not containing $\pi(x)^{\bar{p}}$. With the notation used in Section 3,

$$
b(\bar{p}, \bar{q})=\sum_{R} \frac{\Pi \bar{l}(R) ! Q(R) \mu_{\bar{q}}^{I}(\bar{l}(R))}{\Pi(u(R)) !},
$$

the summation being exactly as in Theorem 3.1 .

5. Applications and Numerical Complexity. In order to apply Theorem 3.1 (or its analogue 4.1), we shall give some data and analyze the complexity of the method with an eye towards applications. Since the numbers $b(\bar{p}, \bar{q}), \bar{q}$ fixed, are calculated in the same way as $s b(\bar{p})$, with the polynomial $\mu_{\bar{q}}(s)$ playing the role of $\mu_{k}(s)$, we shall stick to the $s b$-case and consider monomials $\pi(x)^{\bar{p}}$. Let $n$ be the number of nonzero components of $\bar{p}, k=\frac{1}{2} K(\bar{p})=\frac{1}{2} \sum p_{i}, \max \left\{p_{i}\right\} \leqslant m$, and for convenience denote the different nonzero components of $\bar{p}$ by $p_{1}, p_{2}, \ldots, p_{t}$ with $p_{i}$ occurring $r_{i}$ times in $\bar{p}$. Then we have the following formula of Theorem 3.1,

$$
s b(\bar{p})=\sum_{R} \frac{\Pi \bar{l}(R) ! Q(R) \mu_{k}^{I}(\bar{l}(R))}{\Pi(u(R)) !} .
$$


This formula works for all $\bar{p}$ with the same value of $k$, irrespective of $n$, and $\left\{\mu_{k}^{I}(\bar{l})\right\}$ are the coefficients of a polynomial calculated once and for all. Since we assume $p_{i} \leqslant m$, we use the ideal $I=I(m)$, and the polynomial is chosen to be $\mu_{k} \bmod I(m)$, cf. (2.8). Define

$$
\begin{aligned}
\#\left(\mu_{K} \bmod I(m)\right)= & \text { number of nonzero coefficients of } \\
& \mu_{k} \bmod I(m), \text { cf. Table II; } \\
p_{I}\left(r_{i}, p_{i}\right)= & \text { number of }() \text {-partitions of } r_{i} \text { of } \\
& \text { dimension } p_{m}\left(p_{i}\right), \text { cf. Section } 3 .
\end{aligned}
$$

Then $\Pi h_{I}\left(r_{i}, p_{i}\right)$ is the cardinality of the summation space $\{R\}$ in (1). For each $R$ one needs to calculate the vector $\bar{l}(R)$ and the matrix $u(R)$ of dimension $m \times n$. Furthermore, one must pick out a specific coefficient (corresponding to the exponent $i(R))$ of $\mu_{k} \bmod I(m)$, and this takes (on the average) $\log _{2} \#\left(\mu_{k} \bmod I(m)\right.$ ) operations (assuming the polynomial is available and suitably coded in the computer). Such considerations, together with testing experience, lead us to a reasonably simple estimate of the work needed to calculate $s b(\bar{p})$, namely an "affine" function $\alpha+\beta \cdot N C(\bar{p})$. Here $\alpha, \beta$ are approximately constant (within reasonable bounds of $n, k)$, and

$$
N C(\bar{p})=n m \cdot \log _{2} \#\left\{\mu_{k} \bmod I(m)\right\} \cdot \prod_{i=1}^{t} h_{I}\left(r_{i}, p_{i}\right)
$$

is called the numerical complexity. Such an estimate is of importance when $N C(\bar{p})$ is large, since comparison of relative magnitudes can be very useful. Therefore, we shall also regard $N C(\bar{p})$ as a rough measure of the efficiency (or computing time) of (1).

Recall that monomials equivalent in the sense of Section 1 give the same number $s b(\bar{p})$. Thus the above discussion suggests another definition of root monomial, i.e., "simplest" representative, namely the root should minimize the numerical complexity. It is, indeed, a simple matter to calculate the quantity $N C(\bar{p})$; the factors $p_{I}\left(r_{i}, p_{i}\right)$ are less than 20 in our applications. In most cases, $N C$ is actually minimal for the root $\bar{r}$ chosen in Theorem 1.4. In the exceptional cases it is minimal for one of the closest neighbors of $\bar{r}$ in the tree structure defined by the graphic relation in Section 1 , and then $*(\bar{r})$ seems to be a good choice. There are extreme cases, such as $\bar{r}=(666552222),{ }^{*}(\bar{r})=(666633222)$, where ${ }^{*}(\bar{r})$ will reduce numerical complexity by $40-50 \%$, that is, formula (1) is almost twice as fast when $*(\bar{r})$ is used rather than $\bar{r}$.

Reduction to roots can be useful when some numerical quantity, such as $s b(\bar{p})$, is constant on the equivalence class of $\bar{p}$. The number of unlabelled graphs with vertex degree sequence $\bar{p}$ is another example. Thus, in the table in Read and Wormald [13] about $21 \%$ of the entries are actually redundant (if roots were identified). A computer program can easily generate successively roots of a certain dimension $n$ (cf. Table I, Section 2, $d=n-2$, for $n=15$ the number of roots is about $3 \cdot 10^{6}$ ). The table in [6] contains all roots for $n \leqslant 10$, defined by the algorithm in 1.4.

Suppose one is interested in applying (1) to a large collection $A$ of monomials with fixed $k$. Rather than using one polynomial $\mu_{k}^{I}$, $I$ small, one should calculate several polynomials modulo large ideals $I_{i}$, adapted to a suitable partition $\left\{A_{i}\right\}$ of 
$A$. When $k$ is large, say $k>20$, this seems to be the most economic strategy. Indeed, the generation of these polynomials $\mu_{k}$ presents no serious problem in dimensions where (1) is applicable. However, it is difficult to control the effect of truncation errors in (1), so this may call for multiple-precision arithmetic. Then we propose to calculate the (large) integer coefficients of $\bar{\mu}_{k}=2^{k} k ! \mu_{k}$, since using these in (1) the contribution to the sum in (1) will be integral for each $R$. For the magnitude $10^{e}$ of the largest coefficient of $\bar{\mu}_{k} \bmod I(m)$ the exponent $e$ increases roughly linearly with $k$ and relatively little with $m$. A few examples are

\begin{tabular}{|c|c|c|c|c|c|c|}
\hline$(k, m)$ & $(5,5)$ & $(10,5)$ & $(20,5)$ & $(25,3)$ & $(30,2)$ & $(30,4)$ \\
\hline$e$ & 3 & 8 & 24 & 25 & 32 & 34 \\
\hline
\end{tabular}

However, experience with (1) has shown that $e$ may be well above the number of significant digits representable in the computer. We have performed calculations of $\mu_{k}$ and $s b(\bar{p})$ for $k \leqslant 30$ using a CYBER 171 and standard FORTRAN with double-precision arithmetic (28 decimal digits).

The growth of the numbers $\gamma_{k}=\#\left(\mu_{k} \bmod I(m)\right)$ is shown in Table II, indicating that $\gamma_{k} / \gamma_{k+1}$ decreases and is at most 1.4. Note that in order to calculate $s b(\bar{p})$ for all $\bar{p}$ up to dimension $n$, one needs $\mu_{k}$ for $k$ up to [ $\left.n(n-1) / 4\right]$ and $m$ at most $n-2$ (cf. the discussion of root monomials in Section 1). For example, $k \leqslant 22$ or 27 suffices for $n$ up to 10 or 11 , respectively.

\section{TABLE II}

The number of nonzero coefficients of $\mu_{k} \bmod I(m)$

\begin{tabular}{|r|r|r|r|r|r|r|r|r|}
\hline$k$ & \multicolumn{1}{|r|}{3} & \multicolumn{1}{|c|}{4} & \multicolumn{1}{|c|}{5} & \multicolumn{1}{|c|}{6} & 7 & 8 & \multicolumn{1}{|c|}{$k$} & \multicolumn{1}{|c|}{$2 k$} \\
\hline 5 & 9 & 16 & 17 & & & & 17 & 24 \\
10 & 15 & 31 & 35 & 48 & 49 & & 49 & 64 \\
14 & 26 & 67 & 84 & 132 & 140 & 177 & 203 & 232 \\
18 & 70 & 147 & 211 & 388 & 437 & 614 & 947 & 1040 \\
22 & 100 & 458 & 445 & 937 & 1127 & 1741 & & \\
27 & 145 & 785 & 1617 & 4372 & 2535 & 4276 & & \\
30 & 176 & 1041 & 2297 & 6669 & 9722 & 19158 & & \\
\hline
\end{tabular}

The numerical complexity expression (5.1) tells us how effective the computing algorithm (1) is for a given $\bar{p}$ (if a suitable polynomial $\mu_{k}$ is available). The different factors of the expression give precise quantitative information as to the effect of changing certain characteristics of $\bar{p}$. Thus, we are able to predict the work (time) needed to calculate $s b(\bar{p})$, and hence identify those $\bar{p}$ such that (1) works within reasonable bounds. It is instructive to study the variation of $N C(\bar{p})$ for a fixed $k$ or $n$, and compare average values, worst case situations, etc.

Let $N C_{k}$ be the average numerical complexity for all root monomials $\bar{p}$ such that $k=\frac{1}{2} \sum p_{i}$, where roots are chosen as in Theorem 1.4. Then $N C_{k}$ increases exponentially with $k$. More precisely, based upon data for $k \leqslant 22$, we find the following linear equation

$$
\log _{10}\left(N C_{k}\right)=0.65+(0.388) k
$$


by performing a simple linear regression analysis. [There is, of course, no randomness involved. However, Eq. (3) explains $99.7 \%$ of the variation of $\log \left(N C_{k}\right)$ as a function of $k$.] Although $N C_{k}$ represents the typical magnitude of numerical complexity for root monomials, the complexity can be up to 6-7 times larger in the worst case and very much smaller in the simplest cases (for fixed $k$ ).

If one is interested in producing a complete table of coefficients $s b(\bar{p})$ for all monomials up to a certain dimension, a recursive procedure is probably best, since then the information in lower dimensions can be used during the calculations in the next dimension. However, it was too tempting to run out coefficients $s b(\bar{p})$ for monomials $\pi(x)^{\bar{p}}$ of dimension $n \leqslant 10(k \leqslant 22)$, and, moreover, in dimensions $n \leqslant 20$ with $\max \left\{p_{i}\right\}$ equal to $3,4, \ldots$, to keep the complexity at a reasonable level. A few illustrating examples are listed below, cf. Johnsen and Straume [5].

\section{TABLE III}

The number $s b(\bar{p})$ of symmetric zero-diagonal binary matrices with various row sum $\bar{p}$.

$$
n=\#\left\{j \mid p_{j}>0\right\}, k=\frac{1}{2} \sum p_{i} \text {. }
$$

\begin{tabular}{|r|r|l|l|l|}
\hline$k$ & $n$ & \multicolumn{1}{|c|}{$\bar{p}$} & $N C(\bar{p})$ & \multicolumn{1}{c|}{$s b(\bar{p})$} \\
\hline 2 & 4 & 1111 & 4 & 3 \\
6 & 5 & 33222 & $1.3 \cdot 10^{3}$ & 7 \\
10 & 10 & 5222222111 & $1.6 \cdot 10^{4}$ & 15585 \\
13 & 8 & 55544111 & $3.7 \cdot 10^{5}$ & 6 \\
15 & 14 & 43322222222211 & $1.3 \cdot 10^{5}$ & 3810480099 \\
18 & 9 & 766443321 & $1.1 \cdot 10^{8}$ & 159 \\
20 & 10 & $8765432221^{*}$ & $1.4 \cdot 10^{9}$ & 49 \\
21 & 10 & $8765443221^{*}$ & $3.2 \cdot 10^{9}$ & 399 \\
26 & 13 & $44 \ldots 44$ & $1.2 \cdot 10^{6}$ & 52113376310985 \\
28 & 14 & $44 \ldots 44$ & $1.7 \cdot 10^{6}$ & 6551246596501035 \\
30 & 15 & $44 \ldots 44$ & $2.3 \cdot 10^{6}$ & 945313907253606891 \\
30 & 20 & $33 \ldots 33$ & $1 \cdot 10^{5}$ & 976273961160363172131825 \\
\hline
\end{tabular}

(* indicates the worst case for the given $k$ ).

Institute of Mathematics and Physical Sciences

University of Tromsø

N-9000 Tromsø, Norway 1976.

1. J. A. Bondy \& U. S. R. MURTy, Graph Theory with Applications, American Elsevier, New York,

2. P. ERdOS \& T. GallaI, "Graphs with prescribed degrees of vertices," Mat. Lapok, v. 11, 1960, pp. 264-274. (Hungarian)

3. P. Hanlon, "Enumeration of graphs by degree sequence," J. Graph Theory, v. 3, 1979, pp. 295-299.

4. F. Harary \& E. Palmer, Graphical Enumeration, Academic Press, New York, 1973.

5. B. Johnsen \& E. Straume, Combinatorial Tables I. Evaluation of the Product $\Pi_{i<j}\left(1+x_{i} x_{j}\right)$, Univ. of Tromsø, 1980.

6. B. Johnsen \& E. Straume, Combinatorial Tables II. Graphic Monomials, Univ. of Tromsø, 1981.

7. A. Kerber, Representation of Permutation Groups I, Springer-Verlag, Berlin and New York, 1971.

8. J. LEVINE, "Coefficient identities derived from expansions of elementary symmetric function products in terms of power sums," Duke Math. J., v. 28, 1961, pp. 89-106.

9. P. A. MacMahon, Combinatory Analysis I, Cambridge, 1915.

10. R. C. READ, “The enumeration of locally restricted graphs I," J. London Math. Soc., v. 34, 1959, pp. 417-436. 
11. R. C. READ, “The enumeration of locally restricted graphs II," J. London Math. Soc., v. 35, 1960, pp. 344-351.

12. R. C. READ, "The use of $S$-functions in combinatorial analysis," Canad. J. Math., v. 20, 1968, pp. 808-841.

13. R. C. READ \& N. C. Wormald, Enumeration of Unlabelled Graphs on 6 to 10 Vertices According to Partition, Research Report CORR 79-33, Faculty of Math., Univ. of Waterloo, 1979.

14. R. C. Read \& N. C. Wormald, Counting the 10-Point Graphs by Partition, Research Report CORR 79-42, Faculty of Math., Univ. of Waterloo, 1979.

15. J. RIORDAN, "Symmetric function expansions of power sum products into monomials and their inverses," Duke Math. J., v. 38, 1971, pp. 285-294.

16. H. J. RYSER, Combinatorial Mathematics, Wiley, New York, 1965.

17. E. SNAPPER, "Group characters and nonnegative integral matrices," J. Algebra, v. 19, 1971, pp. 520-535. 